\title{
A SOLUTION ON IDENTIFICATION AND REARING FILES IN SMALLHOLD PIG FARMING
}

\author{
Benhai Xiong ${ }^{1,2, *}$, Runting $\mathrm{Fu}^{3}$, Zhaohui $\mathrm{Lin}^{3}$, Qingyao Luo ${ }^{1,2}$, Liang \\ Yang ${ }^{1,2}$ \\ ${ }^{1}$ Institute of Animal Sciences, Chinese Academy of Agricultural Sciences, Beijing, 100193 \\ ${ }^{2}$ State Key Laboratory of Animal Nutrition, Beijing 100193 \\ ${ }^{3}$ Animal Husbandry and Veterinary Bureau, Tianjin 300210 \\ ${ }^{*}$ Corresponding author, Address: No. 2 Yuanmingyuan West Road Beijing 100193, China, \\ Tel:+86-10-62811680, Fax:+86-10-62815988,Email: bhxiong@iascaas.net.cn
}

Abstract: In order to meet government supervision of pork production safety as well as consumer's right to know what they buy, this study adopts animal identification, mobile PDA reader, GPRS and other information technologies, and put forward a data collection method to set up rearing files of pig in smallhold pig farming, and designs related metadata structures and its mobile database, and develops a mobile PDA embedded system to collect individual information of pig and uploading into the remote central database, and finally realizes mobile links to the a specific website. The embedded PDA can identify both a special pig bar ear tag appointed by the Ministry of Agricultural and a general data matrix bar ear tag designed by this study by mobile reader, and can record all kinds of inputs data including bacterins, feed additives, animal drugs and even some forbidden medicines and submitted them to the center database through GPRS. At the same time, the remote center database can be maintained by mobile PDA and GPRS, and finally reached pork tracking from its origin to consumption and its tracing through turn-over direction. This study has suggested a feasible technology solution how to set up network pig electronic rearing files involved smallhold pig farming based on farmer and the solution is proved practical through its application in the Tianjin's pork quality traceability system construction. Although some individual techniques have some adverse effects on the system running such as GPRS transmitting speed now, these will be resolved with the development of communication technology. The full implementation of the solution around China will supply technical supports in guaranteeing the quality and safety of pork production supervision and meet consumer demand.

Please use the following format when citing this chapter:

Xiong, B., Fu, R., Lin, Z., Luo, Q. and Yang, L., 2009, in IFIP International Federation for Information Processing, Volume 295, Computer and Computing Technologies in Agriculture II, Volume 3, eds. D. Li, Z. Chunjiang, (Boston: Springer), pp. 2249-2258. 
Keywords: Pig; Smallhold Pig Farming; Mobile PDA; Rearing files; Traceability

\section{INTRODUCTION}

Since mad cow disease happened in Britain in 1986 (Gollam, E. et al.,2004), the EU takes the lead in study on traceability of beef cattle and calves, and has established identification and tracing system for cattle and beef(F. Madec et al., 2001) . In Canada, identification management for livestock and poultry is taken as an important part of agricultural development strategy called "Brand Canada" and will cover 80 percent of the whole livestock and poultry and their products until the end of 2008 according to this plan(Brand Canada, 2008). After the first case of mad cow disease occurred in the US in 2003, US government makes a plan for the identification and traceability system of cattle and decides to enforce it in the whole country until 2009 so as to track relevant animals within 48 hours in case of epidemic diseases(USDA, 2005). In June 2006 , the Japanese Congress enacted " the beef production traceability" and made certain to establish a national animal traceability information system. as the act stipulates, all cattle produced in Japan must have their own identification number after birth and information including identification number, date of birth, cattle breed, delivery records each cattle are managed by the Livestock Improvement Center(Chinacity,2008). The same are Australia and the New Zealand. On June 1, 2007, the Ministry of Agriculture promulgated the " the Administrative Rules on identification and rearing files for animal and poultry"(Chinaagri,2008), which has put into effect. Study on key traceability technologies for animal diseases and quality and safety and construction of traceability system have be establishing by various project channels.

On the basis of "the Administrative Rules" enacted by the Ministry of Agriculture(MAPRC,2006) and intelligent reader devices, together with identification information technologies, mobile intelligent technology and network database technology, regard to application in practice and economical characteristic of technologies, through integration of technologies and devices, this study proposes a solution for individual electronic rearing files of pig and corresponding system for smallhold pig farming, and is also demonstrated for application. 


\section{EQUIPMENT AND METHOD}

\subsection{Equipment}

Basis identifying device provided by Beijing PingZhi Dongfang Sci \& Tech Development Limited is ZX-6000 mobile intelligent readers (PZDF, 2008) in which the operation platform is window mobile 5.0 system, its characteristics are as follows: 1) embedded smart IC card reading and writing device, consistent with "China financial integrated circuit IC card and application standards", can read and write contact smart card IC , 2) identifying bar code: integrate a camera with 300 thousands pixels, one third inch of optical specification, one-dimension or two-dimension bar code can be photographed through the camera and identified.

\subsection{Selection of individual identification for pig}

Individual identification for pigs adopts integrated identification mode combining special individual barcode for pig with common barcode. One side adopts barcode ear tag recommended by the Ministry of Agriculture(Fig.1,left) which tag code rules comply to "the Administrative Rules", and detailed contents are described in Xiong benhai et al.(2007) and must select the tag identification devices appointed by the Ministry of Agriculture, including ZhiXing ZX-6000 mobile identification reader(Fig.1,right). The advantage by use of this way is that ear tags could produce and manage in the whole, but its disadvantage is easy to result in monopolization and to affect technologies renovation and to make against application of universal technologies. Therefore, this study develops identification embedded module fro common code on ZX-6000 in order to be able to identify other bar ear tag with other code standards.
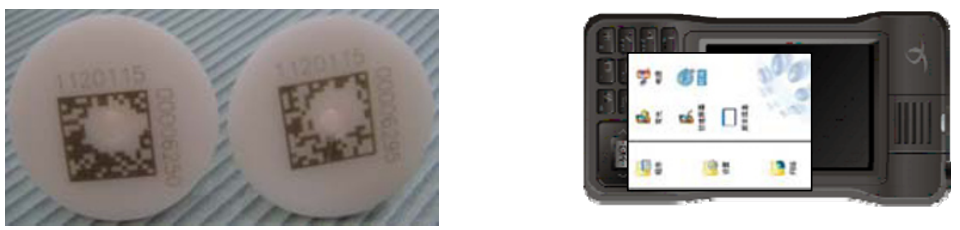

Fig.1 Special pig ear-tag and ZX-6000 PDA

As Fig. 2 shows, the ear tag designed and produced in the study is plastic and conforms to the requirement of the Ministry of agriculture, but its picture bar code uses Data Matrix(DM) code which bears more information in the smaller area(Ec21 cn, 2008). Despite its appearance size is the same as the ear tag in Fig 1, it is improved including: Firstly, the latter uses bolder 
font with legible and concise, and can be identified by naked eyes in case of no reader or disused. Secondly, because the latter adopts short-matrix 2D bar code, area required for bearing the same information can wholly be printed in any side from center dot, and its identification percent could almost achieve $100 \%$, but the ear tag in the Fig. 1 must cover the center dot and has spoiled picture when printed resulting in identifying slowly and sometimes un-identified. Thirdly, from view of the reader, the former can only be identified by appointed ZX -6000, but the later can be identified by allpurpose reader on the right of Fig.2. This study adds common decode programs on ZX-6000 with embedding special identification code in Fig 1 so as to identify DM code (also other generic code). Tag code information identified by using the PDA reader is directly sent into modules of immunity, supervision or tracing in PDA system, that is say identification and data treatment accomplish in the same device, but code data identifying through other general readers need transport into the system by line of USB.
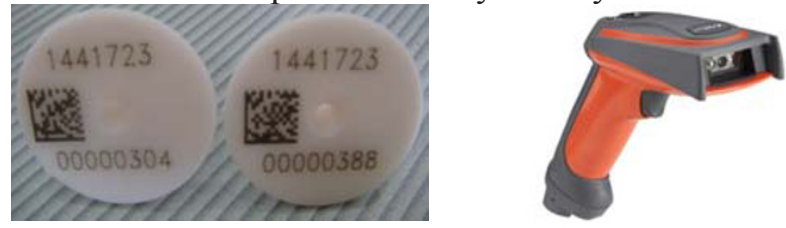

Fig.2 DM-bar ear tag(left) and all purpose bar scanner

\subsection{Information collection and transmission in rearing section}

Identifying pigs and setting electronic rearing files as showed in Fig 3. pigs wearing ear tags in Fig 1 or Fig 2 are identified by the improved mobile intelligent reader, then by the function modules embedded in PDA to collect and to edit information in rearing process, which are directly send to remote traceability database server constructed in the study by GPRS and Internet(Luan Chang-ha et al. 2006), interconnected with information in process of following pig delivery, slaughter or sale or supplied traceability query.

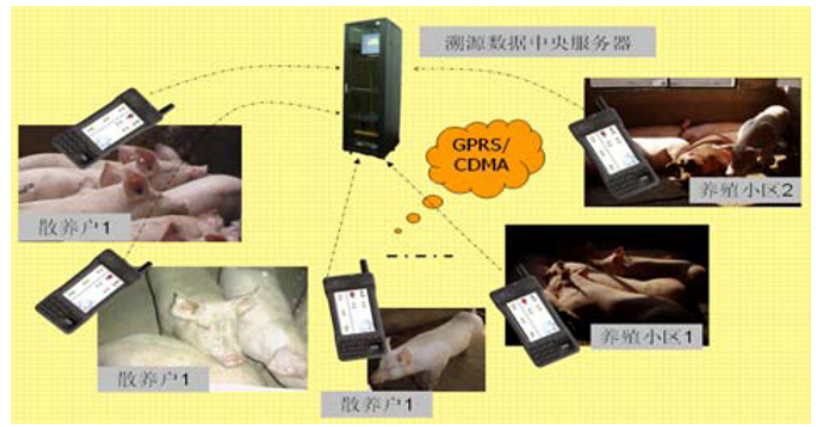

Fig.3 data collection solution of rearing files 


\subsubsection{Design for mete-data items of pig identification and inputs}

Information collection modules in rearing section for swine scattered breeding mainly include setting of ear tag, immunity events, feed events, veterinary events and surveillance events etc.. Design principles of data items reflect primary content of events and obligatory content filled in easily.

As Fig,3, all of rearing information collected by mobile devices from different farmers or breeding districts need to connect with internet through GPRS and then send to traceability center database. But before data storage, information must be reorganized and remove redundant content. Table 1 shows main data fields in the traceability database, of which event fields are used to storage information integrated in the server.

Table.1 The Structure of Tracing Database on Smallhold Farmer mode(tracing database: porktrace.dbo)

\begin{tabular}{clc}
\hline Serial No. & \multicolumn{1}{c}{ Filed Name } & Length \\
\hline 1 & Ear tag & 15 \\
2 & Owner & 10 \\
3 & Breed of pig & 12 \\
4 & Location of breeding & 16 \\
5 & Immunity events & 128 \\
6 & Feed events & 128 \\
7 & Veterinary events & 128 \\
8 & Slaughter events** & 128 \\
9 & Carcass 1* & 20 \\
10 & Carcass 2* & 20 \\
11 & Official Vet & 64 \\
12 & Note & 128 \\
\hline
\end{tabular}

*field content offered by slaughter units, others by breeding owners or farms.

\subsubsection{Development of Embedded PDA System based on rearing section in smallhold pig farming mode}

The embedded system is developed on ZX-6000 mobile PDA reader according to the design in 2.3.1 2.3.2. Developing platform is windows mobile 5.0, and tool software include Visual Studio 2005, Visual C\# 2005, the class libraries run in the mobile device with small memory are .NET Compact Framework(Liu Yan-bo et al. 2006; Fu xi et al.,2005), Database used: is SQL mobile 2005. SQL mobile Client Agent need to be installed on PDA, and Client Agent is communicated with SQL mobile Server Agent through HTTP. Application programming interface for SQL mobile is ADO.net(Fu xi et al.,2005). There are two ways for data storage: Firstly, if signal of wireless GPRS is good, edited data is directly send to traceability center database 
(porktrace.dbo); Secondly, the data is saved by other storage medium when signal is not good.

\section{RESULTS AND DISCUSSION}

\subsection{Setting of ear tags}

As to application of mobile smart traceability system for pig developed in 2.3.2, the first important function is to record ear tag code and to identify ear tags through PDA system as well as recording information about owner, variety and quantity of pigs, dependency etc. In Fig.4, the form shows ear tag ID and basic information of 10 pigs which information is wirelessly send to porktrace.dbo, the traceability database designed according to 2.3.2. Subsequently, the system creates the same quantity of new records in the database making ready for following data processing.

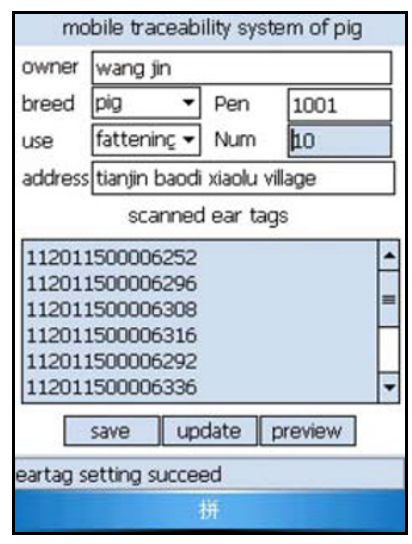

Fig.4 Setting Ear tag by PDA

\subsection{Immunity events}

Fig. 5 shows the record and query result of the pig immunity event. After identifying ear tags through the camera or inputted pig ID, the system displays the Fig. 5 form(left), then input basic immunity information which is directly sent to relevant data records by button of the "Save" and update records of the immunity event in the database. Moreover, the event data can also be remotely viewed and edited by wireless. The right in Fig 5 shows the query result of the immunity event for a certain ear tag. 

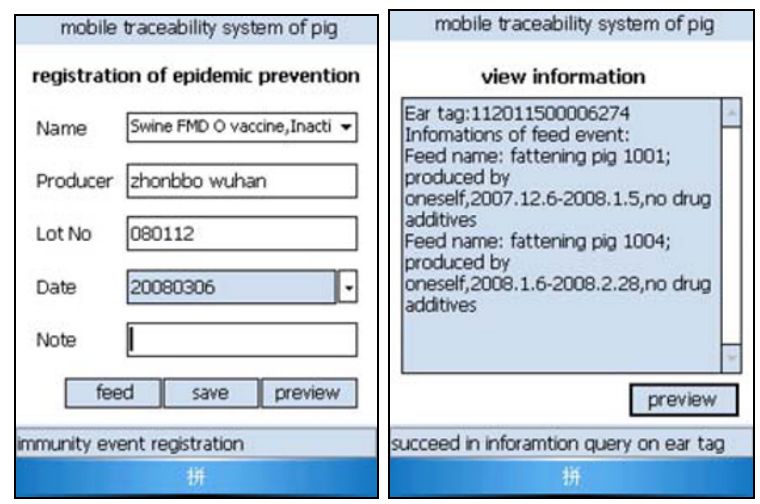

Fig.5 Record immunity event(left) and mobile query (right)

\subsection{Feed events}

Fig 6 shows record and query result of pig feed event. After identifying ear tags through the camera or inputted pig ID, the system displays Fig 6 form, then input basic feed information which is directly sent to relevant data records by the "save" and update records of the feed event in the database. Moreover, the event data can also be remotely viewed and edited by GPRS. The right in Fig 6 shows the query result of the feed event for a certain ear tag, including used feed additives and some drugs to meet their right to know.
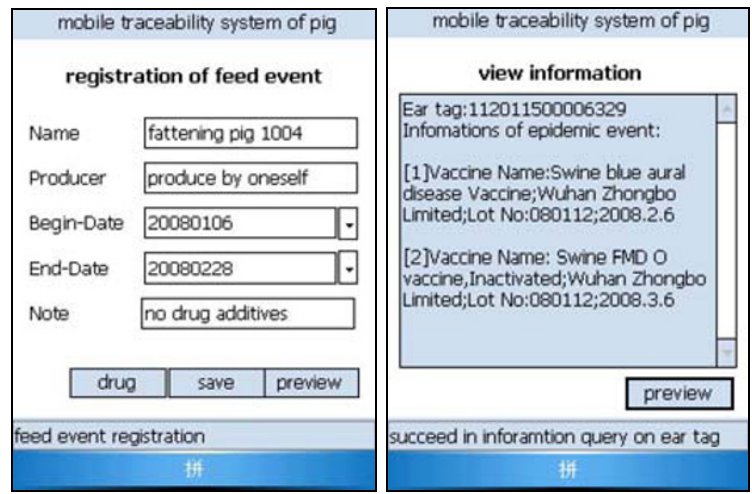

Fig.6 Record Feed event (left) and mobile query(right)

\subsection{Veterinary and Surveillance events}

When outbreak of disease for swine there need to treat diseased pigs with using provided veterinary drug. According to "safe use standards for feed 
additives"(Yang Zhen-hai et al., 2003), 79 kinds of veterinary drugs are allowed to use for the swine in China. The mobile system provides function to record information of used veterinary drugs in breeding process for the treatment of illness. In addition, the mobile PDA system also can record test results by official vet in the breeding sector through module of the surveillance event. At present main testing items in the system include clenbuterol, ractopamine, sulfa drugs and other prohibited drugs. Different from 3.1 3.3, the module is generally provided access for official veterinary not for farmers or consumers.

\subsection{Query results based on Internet}

These ID, inputs and other data collected by the above from 3.1 to 3.4 modules are submitted to remote traceability database, mainly meet to provide information query for consumers after slaughter of pigs with ID and sales in the supermarket. Data query in the study has integrated on traceability query platform that is www.tjporktrace.com or www.porktrace.com.cn built by Xiong Benhai et al. (2007). This study only offers the client query page in Fig 6 to show integrality of the system, but technical details do not discuss in this study.

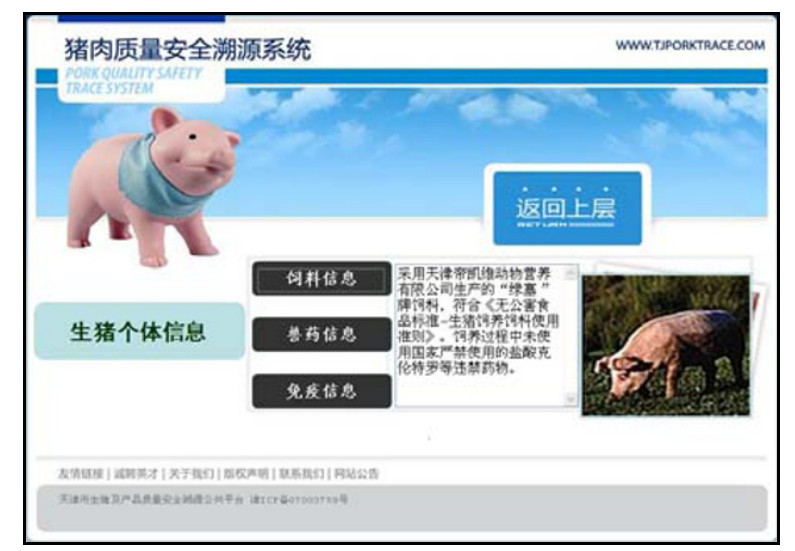

Fig. 6 Web traceability platform (www.tjporktrace.com )

\subsection{Discussion}

Firstly, this solution is a part of whole technical solution in the project of "digital monitor and traceability technologies of pork quality safety in Tianjin city of China", and makes an application in demonstration districts and obtains expected effects. The mobile PDA system can conveniently collect information without restrictions of time and space and provides a feasible 
technical solution for building electric rearing files of a lots of smallhold farmers(Zhang Jing, 2007), also is applied to those scale farms.

Secondly, the running fee for GPRS is not a problem. At present, China Mobile Company provides a monthly package "GPRS mobile Internet five Yuan package with 10 Mbytes mobile Internet flow by a named dynamic zone of the SIM card (BDZGP, 2008). Data contents in the mobile data collection system are mainly text and digital data, even if a scale of pig-farm achieves its data from several hundreds of heads or thousands, data capacity of rearing information is not more than 10 Mbytes in one month. If the installed SIM card in the ZX-6000 reader is only used to collect and send data without functions of calling, the monthly fee will not exceed five People's Yuan.

The third, this study also provides a solution when GPRS does not work. When GPRS can be connected, the system will send the data to the traceability database, otherwise, it storages the information inside a special IC card owned by the PDA firstly and follows to transmit them when GPRS works good.

\section{ACKNOWLEDGEMENTS}

Appreciation is extended to Ma Xing-jian, fromBeijing PingZhi Dongfang Sci \& Tech Development Limited, for his practical suggestions and helps in process of this system development.

\section{REFERENCES}

Beijing D-Zone GPRS Package(BDZGP). http://www.lanxingkong.net/ shoujishang wang/ article.asp?id=28.2008-07-16

Brand Canada. http://www.aquaculture.ca/bcanada.htm :2008-07-18

Chinaagri. http://www.agri.gov.cn/xxlb/t20070319_787945.htm.2008-07-18(in Chinese)

Chinacity.http://www.chinacity.org.cn/cankao/22233_2.html.2008.-07-18(in Chinese)

EC21CN. http://www.ec21cn.org/Education/UploadFiles/CourseWare/tiaoma/tmjc/2/study/53-2.htm.2008-07-18(in Chinese)

F. Madec, R. Geers, P. Vesseur, N. Kjeldsen \& T. Blaha. Traceability in the pig production chain. Rev. sci. tech.Off. int. Epiz., 2001, 20 (2), 523-537.

Fu xi,Qi ning,Xu Jun. Mobile Telephone Application and Development of Windows Mobile $[\mathrm{M}] .2005$ (in Chinese)

Gollam, E. et al. Traceability in the U.S. Food Supply: Economic Theory and Industry Studies. Washington, United States Department of Agriculture,2004. (Agricultural Economic Report Number 830) GS1. Wine Supply Chain Traceability: EAN-UCC Standards Application Guideline. Brussels: 2005.

John D. Lawrence, Daryl Strohbehn, Dan Loy, and Reg Clause. Lessons Learned from the Canadian Cattle Industry:National Animal Identification and The Mad Cow.2003. http://www.card.iastate.edu/publications/DBS/PDFFiles/03mrp7.pdf 
Liu Yan-bo,Hu Yan,Ma qi. Application and Developement of Windows Mobile Platform [M].2006(in Chinese)

Luan Chang-hai,Wang Meng. study on embedded internet design based on GPRS [J]. control \& automation.2006.(22)17,94-96(in Chinese)

Ministraty of Agrilculture of the People's Republic of China(MAPRC).Decree No 67, the Administrative Rules on identification and rearing files for animal and poultry. http://www.agri.gov.cn/blgg/t20060628_638621.htm,June 28,2006(in Chinese)

Ping Zhi Dong Fang(PZDF). http://www.pda9.com/ZX6000.htm.2008-07-18. (in Chinese)

United States Department of Agriculture(USDA), Marketing and Regulatory Programs Animal and Plant Health Inspection Service. National Animal Identification System (NAIS)- Technical Supplement to Draft Program Standards. July 26, 2005.http://animalid.aphis.usda.gov/nais/

Xiong ben hai et al. A practical web-based tracking and traceability information system for the pork products supply chain. New Zealand Journal of Agricultural Research, 2007,Vol.50:725 733.

Yang Zhen-hai,Cai Hui-yi. Standards for safety application of Feedstuff Addtives[M]. Beijing:china agriculture press,2003(in Chinese)

Yu Linhua,Yang yanlin. status and preventing measures of high-pathogenic swine of blue ear disease [J]. HUNAN journal of a science \& veterinary medicine,2007,(3):25-28(in Chinese)

Zhang Jing. Data commentate "force"-Market Analyse for China Swine main production and sale zones in 2006. swine industry science. 2007, 24(1):26 28(in Chinese) 